\title{
Stability, Hopf bifurcation and effects of impulsive antibiotic treatments in a model of drug resistance with conversion delay
}

\author{
Suranath Chomcheon ${ }^{1,2}$, Yongwimon Lenbury ${ }^{1,2^{*}}$ (1) and Warunee Sarika $a^{1,2}$
}

"Correspondence:

Yongwimon.len@mahidol.ac.th ${ }^{1}$ Department of Mathematics, Faculty of Science, Mahidol University, Bangkok, Thailand ${ }^{2}$ Centre of Excellence in

Mathematics, CHE, Ministry of Education, Bangkok, Thailand

\section{Springer}

\begin{abstract}
Drug resistance has become a problem of grave concern for members of the medical profession for many decades. More and more bacterial infections cannot be contained. Therefore it is of imperative importance for us to be able to keep drug resistance under control. Mathematical models can help us to discover possible treatment strategies that could alleviate the problem. In this paper, the dynamic behavior of drug resistance is investigated by studying a model system of differential equations incorporating a delay in the process whereby the sensitive bacteria (bacteria that antibiotics can still attack) is converted into the resistant bacterial strain through plasmid transfers. We give the conditions under which a Hopf bifurcation occurs, leading to a periodic solution. The result indicates that the conversion rate and the delay play a significant role in the development of drug resistance. Also, the impact of periodic antibiotic intakes is taken into account, making the model an impulsive one. Each time a patient takes antibiotics, a fraction $\mu(0<\mu<1)$ of sensitive bacteria dies, but resistant bacteria are left to grow and multiply in periodic bursts. Analysis is carried out on the impulsive system to find the stability criteria for the steady-state solution where bacterial strains are washed out. Numerical simulation is carried out to support our theoretical predictions.
\end{abstract}

Keywords: Drug resistance; Impulsive system; Delayed model; Hopf bifurcation; Periodic solutions

\section{Introduction}

According to [1], emergence of microorganisms resistant to multidrug has become a global concern. The problem is compounded by the fact that resistance pattern of organism not only varies from one country to another but also within the same country. Moreover, "antibiotic resistance is no longer a prediction for the future; it is happening right now, across the world, and is putting at risk the ability to treat common infections in the community and hospitals" [1]. Urgent, coordinated action is crucial for the world to be prepared for the postantibiotic era, in which it will not be as easy to treat or control common infections and minor injuries, as we have been able to do for decades [2]. To complicate matters, the development and spread of antibiotic resistance is multifactorial, so that any studies to try to cope with the situation must as a consequence utilize all possible tools and advanced techniques available. The global evolutionary consequence of antimicrobial utilization is

(c) The Author(s) 2019. This article is distributed under the terms of the Creative Commons Attribution 4.0 International License (http://creativecommons.org/licenses/by/4.0/), which permits unrestricted use, distribution, and reproduction in any medium, provided you give appropriate credit to the original author(s) and the source, provide a link to the Creative Commons license, and indicate if changes were made. 
an extremely complex problem not only intrinsically connected to public health and human functions, but also embroiled in animal health, food production, nutrition, farming, and the environment.

Penicillin was discovered in 1928 by Alexander Fleming, giving rise to a medical revolution whereby a medication and cure of previously life-threatening infections became possible [3]. He also later foretold the risks of antimicrobial resistance (AMR), pointing out the danger that the ignorant man may easily underdose himself and become resistant to it by exposing his microbes to nonlethal quantities of the drug.

Southeast Asia has been proposed as an epicenter for emerging infectious diseases, and that makes the region a focal region for the emergence of AMR due to the fact that it is a highly dynamic region characterized by swift but uneven economic development. It has been reported by the WHO that a lack of systematic collection of data concerning AMR, and associated morbidity, mortality, and economic cost, in Southeast Asia is the risk factor that creates the "burgeoning and often neglected" problem [3]. Surveillance of AMR across Southeast Asia is currently carried out in the healthcare framework, but hospital-associated incidence accounts for only a fraction of the total antimicrobial resistance burden. This has left large gaps in thorough knowledge and understanding on the AMR development and evolution in the human community and in animals cultured for food production.

Apart from data surveillance and analyses, mathematical modeling, for its power of prediction and ability to potentially portray nonlinear dynamic behavior of the process, is able to play a crucial role in improving our understanding of antibiotic resistance. Spicknal et al. [4] review the literature on antibiotic-resistance modeling published between 1993 and 2011. The models' structures are classified into one or more of six categories based on the assumptions made in regards to within-host and population-level competition between antibiotic-sensitive and antibiotic-resistant strains. They explained that each model category has varying dynamic implications in terms of how the use of antibiotic affects resistance persistence, and consequently each may lead to different conclusions about optimal treatment protocols that could minimize resistance. Their paper provides valuable insight into model selection.

To investigate the population dynamics of bacterial infection under antibiotic resistance pressure, in 2007, Puttasontiphot et al. [5] proposed a mathematical model describing the dynamics of the resistant bacterial strain, sensitive strain, and the amount of available nutrients in the gastrointestinal tract, as functions of time. Later, Chayapham and Lenbury [6] considered a nonlinear model of interaction between resistant and sensitive strains of bacteria, extended to account for the delay in the process of plasmid transfer during the sensitivity to resistant conversion $[7,8]$. In this paper, we extend our understanding of AMR by analyzing further the delay differential equation model of drug resistance proposed in [6] for its stability and Hopf bifurcation development. The model is then modified to take into account the impulsive antibacterial drug treatment, which periodically reduces the level of sensitive strain which is susceptible to the drugs, as well as the sudden increases in the resistant strain as the susceptible population being killed by the antibiotics leaves the resistant strain to grow, on the limiting nutrients, and multiply in periodic bursts. Numerical simulations of this model will be conducted to support theoretical predictions. 


\section{Model system}

In 2011, Sirinukunwattana et al. [9] analyzed a model that assumes nutrient abundance and incorporates a delay in drug resistant and wild-type strains interaction, consisting of the following equations:

$$
\begin{aligned}
& \frac{d S}{d t}=\psi_{S} S(t)-H(S(t)) S(t) R(t-\tau)-d_{S} S(t)-K S(t)+I, \\
& \frac{d R}{d t}=\psi_{R}(r-R(t)) R(t-\tau)+H(S(t)) S(t) R(t-\tau)-d_{R} R(t-\tau),
\end{aligned}
$$

where $S$ and $R$ stand for the densities of susceptible and resistant strains, respectively, $\psi_{S} S(t)$ and $\psi_{R}(r-R(t)) R(t-\tau)$ represent the growth rates of susceptible and resistant strains, respectively, $H(S(t)) S(t) R(t-\tau)$ incorporates the bacterial conversion from the susceptible population into the resistant population, $d_{S} S(t)$ and $d_{R} R(t-\tau)$ are the death rates of susceptible and resistant strains, respectively, and $K S(t)$ accounts for the killing rate by administered drugs; $I$ accounts for the constant increase in the susceptible strain due to infection, and $\tau$ is the maturation delay. However, this model did not take into account the delay in the process by which the susceptible bacteria is converted into a resistant bacteria through plasmid transfer, as observed and reported by Andrup et al. [7] and Dostal et al. [8].

Later, in 2013, Chayapham and Lenbury [6] considered a nonlinear model of interaction between resistant and sensitive strains of bacteria, extended later to account for the delay mechanism mentioned in $[7,8]$ resulting in the following model system:

$$
\begin{aligned}
& \frac{d x}{d t}=\frac{a_{1} x(t) z(t)(\gamma-x(t))}{K_{S}+z(t)}-a_{2} x(t)-\frac{\varepsilon_{\gamma} x(t) y(t)}{K_{\gamma}+x(t)}-\omega_{1} x(t), \\
& \frac{d y}{d t}=\frac{\psi_{R} y(t) z(t)}{K_{R}+z(t)}+\frac{\varepsilon_{\gamma} e^{-\mu_{1} \tau} x(t-\tau) y(t-\tau)}{K_{\gamma}+x(t-\tau)}-\omega_{2} y(t), \\
& \frac{d z}{d t}=\left(z^{*}-z(t)\right) \omega_{3}-\frac{a_{3} x(t) z(t)}{K_{S}+z(t)}-\frac{a_{4} y(t) z(t)}{K_{R}+z(t)},
\end{aligned}
$$

where $x(t), y(t)$, and $z(t)$ are the levels of sensitive strain, resistant strain, and limiting nutrient, respectively, with initial condition

$$
\begin{aligned}
& (x(t), y(t), z(t))=\left(\varphi_{1}(t), \varphi_{2}(t), \varphi_{3}(t)\right) \in C_{3}^{+}, \\
& \varphi_{i}(0)>0, \quad i=1,2,3,
\end{aligned}
$$

and $C_{3}^{+}=C\left([-\tau, 0], \Re_{3}^{+}\right)$.

The first term on the right of (1) represents the rate of growth of sensitive strain, which increases with increasing nutrient concentration but saturates to a logistic growth rate when there is an abundance of nutrients $(z \rightarrow \infty)$. The last term in (1), $\omega_{1} x$, is the natural removal rate of sensitive strain, whereas the second term is the death rate of this strain, $a_{1}$ being the death rate constant. The term $\frac{\varepsilon_{\gamma} x(t) y(t)}{K_{\gamma}+x(t)}$ represents the rate at which the sensitive train is converted into the resistant population. This saturates to $\varepsilon_{\gamma} y(t)$ when there is an abundance of the resistant population, $\varepsilon_{\gamma}$ being the conversion constant of variation.

The first term on the right of (2) is the growth rate of resistant strain, which is assumed to be a Monod-type function that saturates to $\psi_{R} y(t)$ when there is an abundance of nutrients 
$(z \rightarrow \infty), \psi_{R}$ being the saturation constant. The second term here is the rate at which the resistant strain increases from conversion of the sensitive strain. The model takes into account the conversion delay $\tau$ by this second term on the right of (2), where $e^{-\mu_{1} \tau}$ takes into account the probability that a resistant bacteria survives from the time $t-\tau$ to the time $t$. Here we assume the simplest possible survival distribution obtained by assuming a constant risk over time, so the hazard $\mu_{1}$ is constant for all time [10]. The corresponding survival function is then $\sigma(t)=e^{-\mu_{1} t}$. The last term on the right of (2) is the removal rate of resistant strain by natural means.

In (3) the term $z^{*} \omega_{3}$ represents the constant rate of increase of nutrients from food intakes. Nutrients are removed by natural means at the rate $\omega_{3} z$, utilized for growth of sensitive strain and the resistant strain according to the second and last terms of (3), respectively.

In [6] the conditions are given that ensure the existence and local asymptotical stability of the bacteria free equilibrium $E^{*}=\left(0,0, z^{*}\right)$, the sensitive bacteria-free equilibrium $E_{0}=$ $\left(0, y_{0}, z_{0}\right)$, and the resistant bacteria-free equilibrium $E_{1}=\left(x_{1}, 0, z_{1}\right)$ of $(1)-(3)$, where

$$
\begin{aligned}
& y_{0}=\frac{\left(z^{*}-z_{0}\right) \omega_{3} \psi_{R}}{a_{4} \omega_{2}}, \\
& z_{0}=\frac{\omega_{2} K_{R}}{\psi_{R}-\omega_{2}}, \\
& x_{1}=\frac{\left(z^{*}-z_{1}\right) \omega_{3}\left(K_{S}+z_{1}\right)}{a_{3} z_{1}}, \\
& z_{1}=\frac{\left(\omega_{1}+a_{2}\right) K_{S}}{a_{1}\left(\gamma-x_{1}\right)-\left(a_{2}+\omega_{1}\right)} .
\end{aligned}
$$

The next theorem [6] provides the conditions for the stability of the endemic equillibrium $E_{2}=\left(x_{2}, y_{2}, z_{2}\right)$, where

$$
\begin{aligned}
& x_{2}=\left[\left(z^{*}-z_{2}\right) \omega_{3}+\frac{a_{4} y_{2} z_{2}}{K_{R}+z_{2}}\right] \frac{K_{S}+z_{2}}{a_{3} z_{2}}, \\
& y_{2}=\left[\frac{a_{1} z_{2}\left(\gamma-x_{2}\right)}{K_{S}+z_{2}}-a_{2}-\omega_{1}\right] \frac{K_{\gamma}+x_{2}}{\varepsilon_{r}}, \\
& z_{2}=\frac{K_{R} \omega_{2} K_{\gamma}+\left(\omega_{2}-\varepsilon_{r} e^{-\mu_{1} \tau}\right) K_{R} x_{2}}{K_{\gamma}\left(\psi_{R}-\omega_{2}\right)+\left(\psi_{R}-\omega_{2}+\varepsilon_{r} e^{-\mu_{1} \tau}\right) x_{2}} .
\end{aligned}
$$

For convenience, we first introduce the following parameters:

$$
\begin{aligned}
& b_{1}=\frac{a_{1} x_{2} z_{2}}{K_{S}+z_{2}}-\frac{\varepsilon_{\gamma} x_{2} y_{2}}{\left(K_{\gamma}+x_{2}\right)^{2}}, \quad b_{2}=\frac{\varepsilon_{\gamma} x_{2}}{K_{\gamma}+x_{2}}, \\
& b_{3}=\frac{a_{1} K_{S} x_{2}\left(\gamma-x_{2}\right)}{\left(K_{S}+z_{2}\right)^{2}}, \quad b_{4}=\frac{\varepsilon_{\gamma} K_{\gamma} y_{2} \sigma}{\left(K_{\gamma}+x_{2}\right)^{2}}, \\
& b_{5}=\frac{\psi_{R} K_{R} y_{2}}{\left(K_{R}+z_{2}\right)^{2}}, \quad b_{6}=\frac{a_{3} z_{2}}{K_{S}+z_{2}}, \\
& b_{7}=\frac{a_{4} z_{2}}{K_{R}+z_{2}}, \quad b_{8}=\frac{a_{3} K_{S} x_{2}}{\left(K_{S}+z_{2}\right)^{2}}+\frac{a_{4} K_{R} y_{2}}{\left(K_{R}+z_{2}\right)^{2}}+\omega_{3}, \\
& B_{1}=b_{1}+b_{8}, \quad B_{2}=b_{1} b_{8}+b_{3} b_{6}+b_{5} b_{7}, \quad B_{3}=\left(b_{3} b_{7}+b_{2} b_{8}\right) b_{4}, \\
& B_{4}=b_{2} b_{4}, \quad B_{5}=\left(b_{1} b_{7}-b_{2} b_{6}\right) b_{5},
\end{aligned}
$$




$$
d_{1}=B_{1}^{2}-2 B_{2}, \quad d_{2}=B_{2}^{2}-2 B_{1} B_{5}-B_{4}^{2}, \quad d_{3}=B_{5}^{2}-B_{3}^{2},
$$

$\sigma$ in (6) being the survival function introduced earlier.

Theorem 1 ([6]) If

$$
\begin{aligned}
& \frac{a_{1} x_{2} z_{2}}{K_{S}+z_{2}}>\frac{\varepsilon_{\gamma} x_{2} y_{2}}{K_{\gamma}+x_{2}}, \\
& d_{1}^{2}-3 d_{2}<0,
\end{aligned}
$$

and

$$
\left(b_{3} b_{7}+b_{2} b_{8}\right) b_{4}+\left(b_{1} b_{7}-b_{2} b_{6}\right) b_{5}>0
$$

then the endemic equilibrium $E_{2}=\left(x_{2}, y_{2}, z_{2}\right)$ is locally asymptotically stable for $\tau \geq 0$.

In the next section, we investigate the possibility of the endemic equilibrium $E_{2}=$ $\left(x_{2}, y_{2}, z_{2}\right)$ losing its stability giving rise to limit cycles emerging from it through a Hopf bifurcation.

\section{Hopf bifurcation}

We now establish the conditions on the system parameters that ensure that a Hopf bifurcation occurs.

The characteristic equation of (1)-(3) at the endemic equilibrium can be written as

$$
\lambda^{3}+B_{1} \lambda^{2}+B_{2} \lambda+\left(B_{3}+B_{4} \lambda\right) e^{-\lambda \tau}+B_{5}=0
$$

where the coefficients are defined in (9). Substituting $\lambda(\tau)=\alpha(\tau)+i \varpi(\tau)$ into (14), we obtain

$$
\begin{aligned}
& (\alpha(\tau)+i \varpi(\tau))^{3}+B_{1}(\alpha(\tau)+i \varpi(\tau))^{2}+B_{2}(\alpha(\tau)+i \varpi(\tau)) \\
& \quad+e^{-\alpha \tau}(\cos \varpi \tau-i \sin \varpi \tau)\left(B_{3}+B_{4}(\alpha(\tau)+i \varpi(\tau))\right)+B_{5}=0 .
\end{aligned}
$$

From the proof of Theorem 1 given in [6] we know that $E_{2}$ is locally asymptotically stable for $\tau=0$, which means that $\alpha(0)<0$. By the continuity of the function $\alpha(\tau)$ we are ensured that $\alpha(\tau)<0$ for values of $\tau$ such that $0<\tau<\tau_{c}$ for some $\tau_{c}>0$. That is, $E_{2}$ remains locally asymptotically stable for these values of $\tau$.

Now suppose that $\alpha\left(\tau_{c}\right)=0$ for some $\tau_{c}>0$ and $\alpha(\tau)<0$ for values of $0<\tau<\tau_{c}$. Then $E_{2}$ may lose its stability at $\tau=\tau_{c}$, where $\lambda(\tau)=i \varpi\left(\tau_{c}\right)$. However, $i \varpi$ is a solution of (14) if and only if

$$
-i \varpi^{3}-B_{1} \varpi^{2}+i B_{2} \varpi+\left(B_{3}+B_{4} i \varpi\right)(\cos \varpi \tau-i \sin \varpi \tau)+B_{5}=0 .
$$

Equating the real and imaginary parts in (16) to zero, we obtain

$$
\begin{aligned}
& B_{1} \varpi^{2}-B_{5}=B_{3} \cos \varpi \tau+B_{4} \varpi \sin \varpi \tau, \\
& -\varpi^{3}+B_{2} \varpi=-B_{4} \varpi \cos \omega \tau+B_{3} \sin \varpi \tau .
\end{aligned}
$$


Squaring and adding (17) and (18), we obtain

$$
\left(B_{1} \varpi^{2}-B_{5}\right)^{2}+\left(B_{2} \varpi-\varpi^{3}\right)^{2}=B_{3}^{2}+B_{4}^{2} \varpi^{2} .
$$

Letting $\theta=\varpi^{2}$ in (19) leads to the equation in $\theta$ as follows:

$$
H(\theta) \equiv \theta^{3}+d_{1} \theta^{2}+d_{2} \theta+d_{3}=0,
$$

where $d_{1}, d_{2}$, and $d_{3}$ are as defined in (10).

To prove our result, we state the following lemma based on [11].

Lemma 1 Let $\theta^{*}=\frac{-d_{1}+\sqrt{d_{1}^{2}-3 d_{2}}}{3}$. If $d_{2}<0$ and $H\left(\theta^{*}\right)<0$, then (20) has at least one positive solution.

Proof Since $d_{3}>0, H(0)=d_{3}>0$. To locate the critical points of $H(\theta)$, we equate the derivative of $H(\theta)$ to 0 to obtain

$$
3 \theta^{2}+2 d_{1} \theta+d_{2}=0
$$

whose roots are $\theta_{1,2}=\frac{-d_{1} \pm \sqrt{d_{1}^{2}-3 d_{2}}}{3}$. If $d_{2}<0$, then we have

$$
\theta^{*}=\frac{-d_{1}+\sqrt{d_{1}^{2}-3 d_{2}}}{3}>0
$$

But, $H\left(\theta^{*}\right)<0$ and $H(\theta) \rightarrow \infty$ as $\theta \rightarrow \infty$. This means that the graph of $H(\theta)$ crosses the positive horizontal axis at least once. Therefore $H(\theta)$ has at least one positive solution.

We can see that if the conditions in Lemma 1 hold, then equation (20) has at least one positive solution. Depending on the values of $d_{i}, i=1,2,3$, (20) can have up to three positive solutions. Without loss of generality, the positive solutions of equation (20) may be denoted by $\theta_{1}, \theta_{2}$, and $\theta_{3}$. Then, writing $\varpi_{i}=\sqrt{\theta_{i}}, i=1,2,3$, and substituting $\varpi=\varpi_{i}$ in equations (17) and (18), we obtain

$$
\begin{aligned}
& B_{1} \varpi_{i}^{2}-B_{5}=B_{3} \cos \varpi_{i} \tau+B_{4} \varpi_{i} \sin \varpi_{i} \tau, \\
& -\varpi_{i}^{3}+B_{2} \varpi_{i}=-B_{4} \varpi_{i} \cos \varpi_{i} \tau+B_{3} \sin \varpi_{i} \tau .
\end{aligned}
$$

Solving for $\tau$,

$$
\frac{B_{1} \varpi_{i}^{2}-B_{5}}{-\varpi_{i}^{3}+B_{2} \varpi_{i}}=\frac{B_{3} \cos \varpi_{i} \tau+B_{4} \varpi_{i} \sin \varpi_{i} \tau}{-B_{4} \varpi_{i} \cos \varpi_{i} \tau+B_{3} \sin \varpi_{i} \tau}
$$

which yields

$$
\tan \varpi_{i} \tau=\frac{\left(B_{1} \varpi_{i}^{2}-B_{5}\right) B_{4} \varpi_{i}-B_{3}\left(\varpi_{i}^{3}-B_{2} \varpi_{i}\right)}{\left(B_{1} \varpi_{i}^{2}-B_{5}\right) B_{3}+B_{4} \varpi_{i}\left(\varpi_{i}^{3}-B_{2} \varpi_{i}\right)}, \quad i=1,2,3 .
$$

Therefore

$$
\tau_{i}^{(n)}=\frac{1}{\varpi_{i}} \tan ^{-1}\left[\frac{\left(B_{1} w_{i}^{2}-B_{5}\right) B_{4} \varpi_{i}-B_{3}\left(\varpi_{i}^{3}-B_{2} \varpi_{i}\right)}{\left(B_{1} \varpi_{i}^{2}-B_{5}\right) B_{3}+B_{4} \varpi_{i}\left(\varpi_{i}^{3}-B_{2} \varpi_{i}\right)}\right]+\frac{2 \pi(n-1)}{\varpi_{i}},
$$

where $i=1,2,3$ and $n=1,2,3, \ldots$. 
Let $\tau_{c}>0$ denote the smallest of such $\tau$, namely

$$
\tau_{c}=\min \left\{\tau_{i}^{(n)}>0,1<i<3, n \geq 1\right\}
$$

and denote by $\varpi_{c}$ the corresponding value of $\varpi$ at this point. We will show that a Hopf bifurcation occurs by establishing that

$$
\left.\frac{d(\operatorname{Re} \lambda)}{d \tau}\right|_{\tau=\tau_{c}} \neq 0
$$

To accomplish this, we further introduce for convenience the following parameters:

$$
\begin{aligned}
& k_{1}=B_{4} \varpi_{c} \tau_{c}, \quad k_{2}=B_{4}-B_{3} \tau_{c}, \\
& \Omega_{1}=B_{2}-3 \varpi_{c}^{2}-k_{1} \sin \varpi_{c} \tau_{c}+k_{2} \cos \omega_{c} \tau_{c}, \\
& \Omega_{2}=2 B_{1} \varpi_{c}-k_{1} \cos \varpi_{c} \tau_{c}-k_{2} \sin \varpi_{c} \tau_{c}, \\
& \Omega_{3}=\mu_{1} B_{4} \varpi_{c}+B_{3} \varpi_{c}, \\
& \Omega_{4}=B_{4} \varpi_{c}^{2}-\mu_{1} B_{3}, \\
& W_{1}=\Omega_{1} \Omega_{3}+\Omega_{2} \Omega_{4}, \quad W_{2}=\Omega_{2} \Omega_{3}-\Omega_{1} \Omega_{4} .
\end{aligned}
$$

Theorem 2 For the critical time lag $\tau_{c}$ and the corresponding $\varpi_{c}$, suppose the conditions in Lemma 1 hold. Also, suppose the following conditions are satisfied:

(i) $\Omega_{1} \neq 0$;

(ii) $\Omega_{2} \neq 0$;

(iii) $W_{1} \sin \varpi_{c} \tau_{c}+W_{2} \cos \varpi_{c} \tau_{c} \neq 0$.

Then (22) holds, and a Hopf bifurcation occurs as $\tau$ increases passed $\tau_{c}$.

Proof Equating real and imaginary parts to zero in (15), we obtain

$$
\begin{aligned}
& \alpha^{3}-3 \alpha \varpi^{2}+B_{1} \alpha^{2}-B_{1} \varpi^{2}+B_{5}+B_{2} \alpha \\
& \quad+e^{-\alpha \tau}\left(B_{4} \varpi \sin \varpi \tau+\left(B_{3}+B_{4} \alpha\right) \cos \varpi \tau\right)=0, \\
& 3 \alpha^{2} \varpi-\varpi^{3}+2 B_{1} \alpha \varpi+B_{2} \varpi+e^{-\alpha \tau}\left(B_{4} \varpi \cos \varpi \tau-\left(B_{3}+B_{4} \alpha\right) \sin \varpi \tau\right)=0,
\end{aligned}
$$

where the coefficients $B_{i}, i=1,2, \ldots, 5$, are as defined in (5)-(10).

Differentiating (25) with respect to $\tau$ and evaluating at $\tau=\tau_{c}$, we obtain

$$
\begin{aligned}
\left.\left(B_{2}-3 \varpi_{c}^{2}\right) \frac{d \alpha}{d \tau}\right|_{\tau=\tau_{c}}-\left.2 B_{1} \varpi_{c} \frac{d \varpi}{d \tau}\right|_{\tau=\tau_{c}} \\
=\left.\left[\left(B_{4} \varpi_{c} \tau_{c}\right) \sin \varpi_{c} \tau_{c}-\left(B_{4}-B_{3} \tau_{c}\right) \cos \varpi_{c} \tau_{c}\right] \frac{d \alpha}{d \tau}\right|_{\tau=\tau_{c}} \\
-\left.\left[\left(B_{4} \varpi_{c} \tau_{c}\right) \cos \varpi_{c} \tau_{c}+\left(B_{4}-B_{3} \tau_{c}\right) \sin \varpi_{c} \tau_{c}\right] \frac{d \varpi}{d \tau}\right|_{\tau=\tau_{c}} \\
+\left[\left(\mu_{1} B_{4} \varpi_{c}+B_{3} \varpi_{c}\right) \sin \varpi_{c} \tau_{c}-\left(B_{4} \varpi_{c}^{2}-\mu_{1} B_{3}\right) \cos \varpi_{c} \tau_{c}\right] .
\end{aligned}
$$


Here we have used the fact that $\alpha\left(\tau_{c}\right)=0$, so that

$$
\left.\frac{d \alpha^{3}}{d \tau}\right|_{\tau=\tau_{c}}=\left.3 \alpha^{2} \frac{d \alpha}{d \tau}\right|_{\tau=\tau_{c}}=0
$$

whereas $\sigma=e^{-\mu_{1} \tau}$, which is the survival function, appears in $B_{3}$ and $B_{4}$, so that

$$
\left.\frac{d B_{3}}{d \tau}\right|_{\tau=\tau_{c}}=\left.\frac{d B_{3}}{d \sigma} \frac{d \sigma}{d \tau}\right|_{\tau=\tau_{c}}=-\left.\mu_{1} B_{3}\right|_{\tau=\tau_{c}}
$$

and similarly for $B_{4}$.

Equivalently, (27) can be written as

$$
\left.\Omega_{1} \frac{d \alpha}{d \tau}\right|_{\tau=\tau_{c}}-\left.\Omega_{2} \frac{d \varpi}{d \tau}\right|_{\tau=\tau_{c}}=\Omega_{3} \sin \varpi_{c} \tau_{c}-\Omega_{4} \cos \varpi_{c} \tau_{c}
$$

using (23). Similarly, from equation (26) we have

$$
\begin{aligned}
\left(B_{2}-\right. & \left.3 \varpi_{c}^{2}\right)\left.\frac{d \varpi}{d \tau}\right|_{\tau=\tau_{c}}+\left.2 B_{1} \varpi_{c} \frac{d \alpha}{d \tau}\right|_{\tau=\tau_{c}} \\
= & {\left.\left[\left(B_{4} \varpi_{c} \tau_{c}\right) \sin \varpi_{c} \tau_{c}-\left(B_{4}-B_{3} \tau_{c}\right) \cos \varpi_{c} \tau_{c}\right] \frac{d \varpi}{d \tau}\right|_{\tau=\tau_{c}} } \\
& +\left.\left[\left(B_{4} \varpi_{c} \tau_{c}\right) \cos \varpi_{c} \tau_{c}+\left(B_{4}-B_{3} \tau_{c}\right) \sin \varpi_{c} \tau_{c}\right] \frac{d \alpha}{d \tau}\right|_{\tau=\tau_{c}} \\
& +\left[\left(\mu_{1} B_{4} \varpi_{c}+B_{3} \varpi_{c}\right) \cos \varpi_{c} \tau_{c}+\left(B_{4} \varpi_{c}^{2}-\mu_{1} B_{3}\right) \sin \varpi_{c} \tau_{c}\right]
\end{aligned}
$$

or, equivalently,

$$
\left.\Omega_{1} \frac{d \varpi}{d \tau}\right|_{\tau=\tau_{c}}+\left.\Omega_{2} \frac{d \alpha}{d \tau}\right|_{\tau=\tau_{c}}=\Omega_{4} \sin \varpi_{c} \tau_{c}+\Omega_{3} \cos \varpi_{c} \tau_{c}
$$

using (23). Eliminating $\left.\frac{d \varpi}{d \tau}\right|_{\tau=\tau_{c}}$ from (28) and (30) and solving for $\left.\frac{d \alpha}{d \tau}\right|_{\tau=\tau_{c}}$, we obtain

$$
\left.\left(\Omega_{1}^{2}+\Omega_{2}^{2}\right) \frac{d \alpha}{d \tau}\right|_{\tau=\tau_{c}}=\left(\Omega_{1} \Omega_{3}+\Omega_{2} \Omega_{4}\right) \sin \varpi_{c} \tau_{c}+\left(\Omega_{2} \Omega_{3}-\Omega_{1} \Omega_{4}\right) \cos \varpi_{c} \tau_{c} .
$$

Therefore we have

$$
\left.\frac{d \alpha}{d \tau}\right|_{\tau=\tau_{c}}=\frac{W_{1} \sin \varpi_{c} \tau_{c}+W_{2} \cos \varpi_{c} \tau_{c}}{\Omega_{1}^{2}+\Omega_{2}^{2}}
$$

using (24). Thus by conditions (i)-(iii) we obtain $\left.\frac{d \alpha}{d \tau}\right|_{\tau=\tau_{c}} \neq 0$.

Hence a Hopf bifurcation occurs when $\tau$ passes through the critical value $\tau_{c}$, and this completes the proof.

In Fig. 1, we show a numerical simulation of (1)-(3), in which the parameters satisfy the conditions in Theorem 2 and $\tau>\tau_{c}$. The solution trajectory, seen here projected onto the $(x, y)$-plane, is observed here to tend toward a limit cycle surrounding the endemic 
Figure 1 Solution trajectory. Numerical simulation of the model system (1)-(3) showing solution trajectory, projected onto the $(x, y)$-plane, tending toward a limit cycle surrounding the endemic equilibrium point. Here, $x(0)=0.3, y(0)=0.4$, $z(0)=0.5, a_{1}=2.407407, a_{2}=0.023077$, $a_{3}=0.00196, a_{4}=0.0007, \omega_{1}=0.7, \omega_{2}=0.7$, $\omega_{3}=0.7 K_{s}=0.7, K_{\gamma}=4.3, K_{R}=5.0, \varepsilon_{r}=0.9, \gamma=4.0$, $\mu=0.01, z^{*}=5.0$, and $\psi_{R}=1.2$

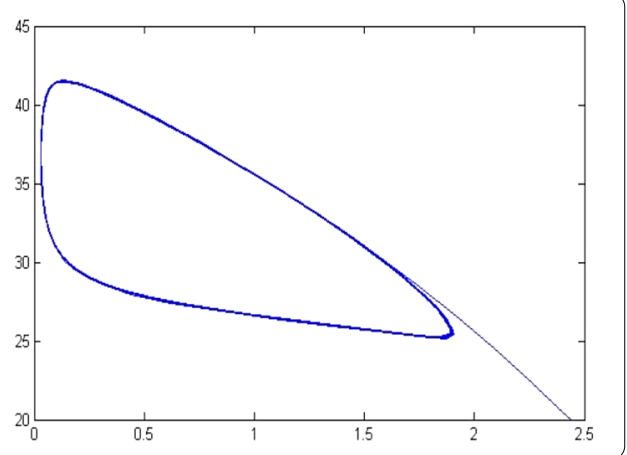

equilibrium as time progresses. The corresponding time series of the sensitive strain $x(t)$, resistant strain $y(t)$, and nutrient concentration $z(t)$ are shown in Fig. 2. It is well known that the amplitude of such a bifurcating periodic solution grows with $\sqrt{|\tau|}$ (see [12] for the details of how the constant of variation in the relationship between the amplitude and $\tau$ can be derived).

In the next section, we consider the impact of periodic drug treatments on the development of resistance to discover how the drug's killing efficacy and the time period between treatments could be appropriately tuned to possibly influence the dynamic behavior of the bacterial interaction under AMR pressure.

\section{Impulsive drug intakes}

We incorporate the effect of a treatment protocol, in which drugs are usually prescribed in a periodic fashion by considering the following impulsive system of delayed differential equations:

$$
\begin{aligned}
& \frac{d x}{d t}=\frac{a_{1} x(t) z(t)(\gamma-x(t))}{K_{S}+z(t)}-a_{2} x(t)-\frac{\varepsilon_{r} x(t) y(t)}{k_{\gamma}+x(t)}-\omega_{1} x(t), \\
& \frac{d y}{d t}=\frac{\psi_{R} y(t) z(t)}{K_{R}+z(t)}+\frac{\varepsilon_{r} e^{-\mu_{1} \tau} x(t-\tau) y(t-\tau)}{k_{\gamma}+x(t-\tau)}-\omega_{2} y(t), \\
& \frac{d z}{d t}=\omega_{3} z^{*}-\frac{a_{3} x(t) z(t)}{K_{s}+z(t)}-\frac{a_{4} y(t) z(t)}{K_{R}+z(t)}-\omega_{3} z \\
& x\left(t_{n}^{+}\right)=(1-r) x\left(t_{n}\right), \\
& y\left(t_{n}^{+}\right)=(1+p) y\left(t_{n}\right), \quad t_{n}=n T, n=1,2, \ldots, \\
& z\left(t_{n}^{+}\right)=z\left(t_{n}\right),
\end{aligned}
$$

$(x(t), y(t), z(t))=\left(\varphi_{1}(t), \varphi_{2}(t), \varphi_{3}(t)\right) \in C_{3}^{+}, \varphi_{i}(0)>0, i=1,2,3$, where $C_{3}^{+}=C\left([-\tau, 0], \Re_{3}^{+}\right)$, and $x\left(t^{+}\right), y\left(t^{+}\right)$, and $z\left(t^{+}\right)$are the right limits of $x(t), y(t)$, and $z(t)$ at time $t$, respectively.

Here $T$ is the period of drug treatments, and $r, 0<r<1$, represents the drug's efficacy in killing the susceptible strain, whereas the positive constant $p$ represents the sudden multiple increase in the resistant strain since the sensitive members being killed by the antibiotics leaves the resistant strain to grow and multiply at a burst on the more readily available nutrients. It is possible for $p$ to be bigger than 1 , considering that the resistant bacterial population may multiply very quickly and each member can reproduce many progenies at a time.

The global existence and uniqueness of solution to the model system (32)-(33) are guaranteed by the smoothness properties of the right-hand sides of the system equations (32)(33) based on [13]. 


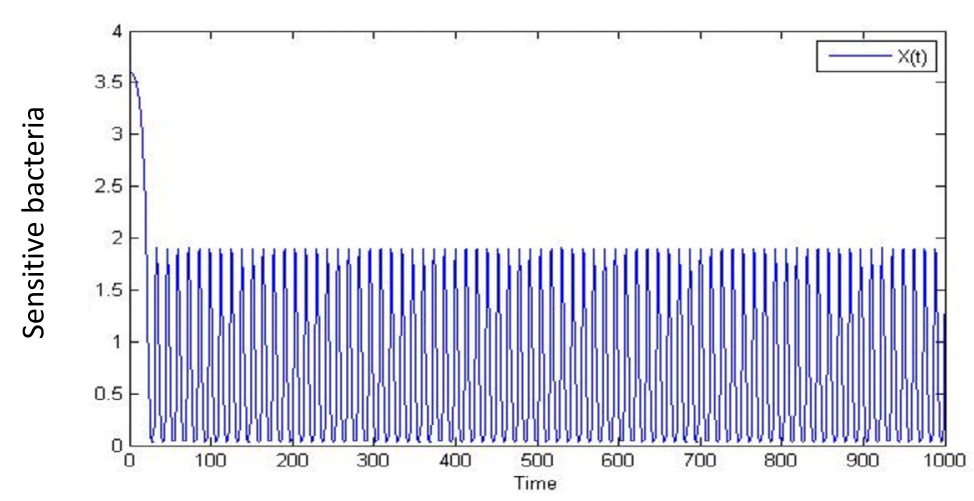

a)

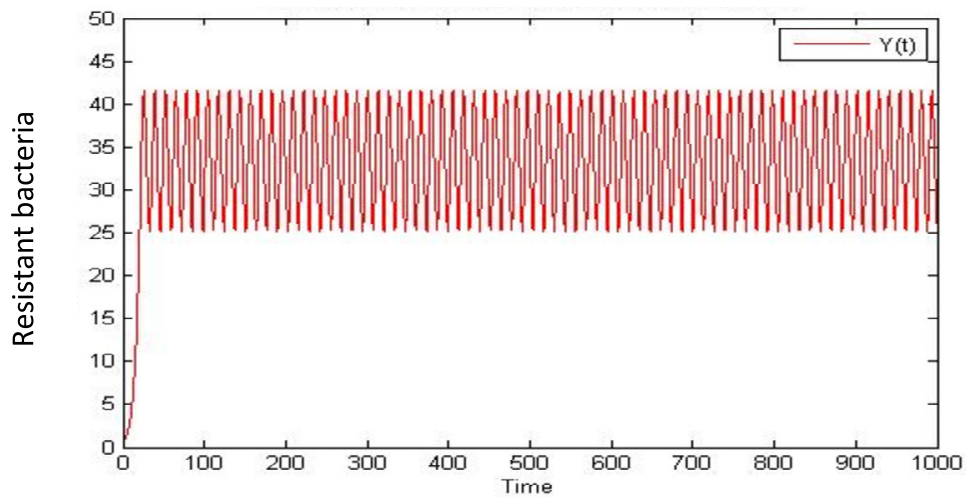

b)

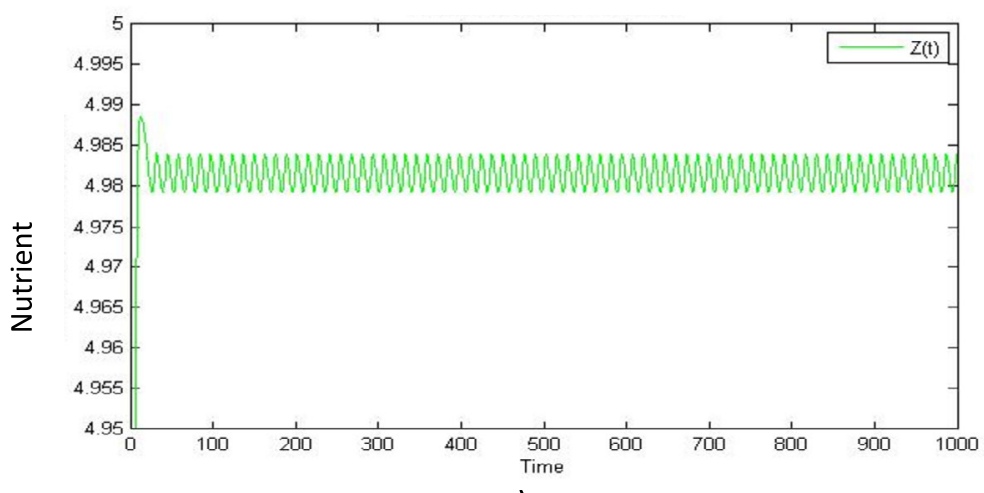

c)

Figure 2 Time series. Numerical simulation of our model showing the time series of (a) level of sensitive bacteria, (b) level of resistant bacteria, and (c) nutrient concentration, corresponding to the case seen in Fig. 1. Here, $x(0)=0.3, y(0)=0.4, z(0)=0.5, a_{1}=2.407407, a_{2}=0.023077, a_{3}=0.00196, a_{4}=0.0007, \omega_{1}=0.7$, $\omega_{2}=0.7, \omega_{3}=0.7 K_{s}=0.7, K_{\gamma}=4.3, K_{R}=5.0, \varepsilon_{r}=0.9, \gamma=4.0, \mu=0.01, z^{*}=5.0$, and $\psi_{R}=1.2$

We can show the following result in a straight forward manner.

Lemma 2 (Nonnegativity) Suppose $(x(t), y(t), z(t))$ is a solution of $(32)-(33)$ with $\varphi_{i}(0) \geq 0$, $i=1,2$, 3. Then $x(t) \geq 0, y(t) \geq 0$, and $z(t) \geq 0$ for all $t \geq 0$. 
Proof Suppose $x(t)$ (or $y(t)$, or $z(t)$ ) becomes negative at some point. Then there must be $t^{*}>0$ such that $x\left(t^{*}\right)=0$ (or $y\left(t^{*}\right)=0$, or $z\left(t^{*}\right)=0$ ) and $\left.\frac{d x}{d t}\right|_{t=t^{*}}<0$ (or $\left.\frac{d y}{d t}\right|_{t=t^{*}}<0$, or $\left.\left.\frac{d z}{d t}\right|_{t=t^{*}}<0\right)$.

Let $t_{1}^{*}$ be the smallest such $t^{*}$, so that $x\left(t_{1}^{*}\right)=0$ and $x(t)>0$ for all $0<t<t_{1}^{*}$. However, when $x=0, t \neq n T$, the first equation of (32) gives

$$
\left.\frac{d x}{d t}\right|_{t=t_{1}^{*}}=0
$$

which contradicts the condition that $\left.\frac{d x}{d t}\right|_{t=t^{*}}<0$. Also, $r<1$. Therefore we have $x(t) \geq 0$ for all $t$.

Next, let $t_{2}^{*}$ be the smallest of the $t^{*}$ such that $y\left(t_{2}^{*}\right)=0$ and $y(t)>0$ for all $0<t<t_{2}^{*}$. However, when $y=0, t \neq n T$, the second equation of (32) gives

$$
\left.\frac{d y}{d t}\right|_{t=t_{2}^{*}}=\frac{\varepsilon_{r} e^{-\mu_{1} \tau} x\left(t_{2}^{*}-\tau\right) y\left(t_{2}^{*}-\tau\right)}{k_{\gamma}+x\left(t_{2}^{*}-\tau\right)}
$$

which is nonnegative because of the definition of $t_{2}^{*}$, and $x$ being non-negative. This contradicts the condition that $\left.\frac{d y}{d t}\right|_{t=t^{*}}<0$. Also, $p>0$. Therefore, we have $y(t) \geq 0$ for all $t$.

Finally, let $t_{3}^{*}$ be the smallest of the $t^{*}$ such that $z\left(t_{3}^{*}\right)=0$, and $z(t)>0$ for all $0<t<t_{3}^{*}$. However, when $z=0, t \neq n T$, the third equation of (32) gives

$$
\left.\frac{d z}{d t}\right|_{t=t_{3}^{*}}=\omega_{3} z^{*}>0
$$

This is a contradiction. Hence, we have $x(t) \geq 0, y(t) \geq 0$, and $z(t) \geq 0$ for all $t \geq 0$. This completes the proof.

We note that system (32)-(33) has the same equilibrium solutions as (1)-(3) whenever they exist and now determine the local asymptotic stability of the bacterial-free solution $\left(0,0, z^{*}\right)$ of system (32)-(33). This is the desirable outcome, a situation where no bacteria persist in the system. To do this, we let

$$
\begin{aligned}
& T_{1}=\frac{K_{S}+z^{*}}{a_{1} \gamma z^{*}-\left(K_{S}+z^{*}\right)\left(a_{2}+\omega_{1}\right)} \log \frac{1}{(1-\mu)}, \\
& T_{2} \equiv \frac{K_{R}+z^{*}}{\omega_{2} K_{R}-\left(\psi_{R}-\omega_{2}\right) z^{*}} \log (1+p) .
\end{aligned}
$$

Theorem 3 Suppose $T_{1}>T_{2}$ and

$$
\begin{aligned}
& \frac{a_{1} \gamma z^{*}}{K_{S}+z^{*}}-a_{2}-\omega_{1}>0, \\
& \frac{\psi_{R} z^{*}}{K_{R}+z^{*}}-\omega_{2}<0, \\
& a_{1} \gamma z^{*}-a_{2}-\omega_{1}+\omega_{3} \neq 0, \\
& \frac{\psi_{R} z^{*}}{K_{R}+z^{*}}-\omega_{2}+\omega_{3} \neq 0 .
\end{aligned}
$$


Then the bacterial-free solution $\left(0,0, z^{*}\right)$ of system (32)-(33) is locally asymptotically stable for $\tau \geq 0$, provided that

$$
T_{1}>T>T_{2} .
$$

Proof The local stability of the solution $\left(0,0, z^{*}\right)$ may be determined by considering the behavior of small amplitude perturbations of this solution. If we define

$$
x(t)=s(t), \quad y(t)=r(t), \quad z(t)=v(t)+z^{*},
$$

then we obtain the following linearized system:

$$
\left[\begin{array}{c}
s^{\prime}(t) \\
r^{\prime}(t) \\
v^{\prime}(t)
\end{array}\right]=\left[\begin{array}{ccc}
\frac{a_{1} \gamma z^{*}}{K_{S}+z^{*}}-a_{2}-\omega_{1} & 0 & 0 \\
0 & \frac{\psi_{R} z^{*}}{K_{R}+z^{*}}-\omega_{2} & 0 \\
\frac{-a_{3} z^{*}}{K_{S}+z^{*}} & \frac{-a_{4} z^{*}}{K_{R}+z^{*}} & -\omega_{3}
\end{array}\right]\left[\begin{array}{l}
s(t) \\
r(t) \\
v(t)
\end{array}\right] .
$$

The corresponding characteristic equation is given by

$$
\left(\frac{a_{1} \gamma z^{*}}{K_{S}+z^{*}}-a_{2}-\omega_{1}-\lambda\right)\left(\frac{\psi_{R} z^{*}}{K_{R}+z^{*}}-\omega_{2}-\lambda\right)\left(\omega_{3}+\lambda\right)=0 .
$$

Consequently, the eigenvalues are

$$
\begin{aligned}
& \lambda_{1}=\frac{a_{1} \gamma z^{*}}{K_{S}+z^{*}}-a_{2}-\omega_{1}, \\
& \lambda_{2}=\frac{\psi_{R} z^{*}}{K_{R}+z^{*}}-\omega_{2}, \\
& \lambda_{3}=-\omega_{3} .
\end{aligned}
$$

The eigenvectors corresponding to the eigenvalues $\lambda_{1}, \lambda_{2}$, and $\lambda_{3}$ are $(1,0, l),(0,1, m)$, and $(0,0,1)$, respectively, where

$$
l=-\frac{a_{3} z^{*}}{\left(K_{S}+z^{*}\right)\left(\omega_{3}+\frac{a_{1} \gamma z^{*}}{K_{S}+z^{*}}-a_{2}-\omega_{1}\right)}
$$

and

$$
m=-\frac{a_{4} z^{*}}{\left(K_{R}+z^{*}\right)\left(\omega_{3}+\frac{\psi_{R} z^{*}}{K_{R}+z^{*}}-\omega_{2}\right)}
$$

which are defined since (38) and (39) hold.

Letting

$$
P=\left[\begin{array}{lll}
1 & 0 & 0 \\
0 & 1 & 0 \\
l & m & 1
\end{array}\right]
$$


and

$$
L_{1}(t)=\left[\begin{array}{ccc}
e^{\lambda_{1} t} & 0 & 0 \\
0 & e^{\lambda_{2} t} & 0 \\
0 & 0 & e^{-\omega_{3} t}
\end{array}\right]
$$

the fundamental solution matrix of (32) is then given by

$$
\phi(t)=P L_{1}(t)=\left[\begin{array}{lll}
1 & 0 & 0 \\
0 & 1 & 0 \\
l & m & 1
\end{array}\right]\left[\begin{array}{ccc}
e^{\lambda_{1} t} & 0 & 0 \\
0 & e^{\lambda_{2} t} & 0 \\
0 & 0 & e^{-\omega_{3} t}
\end{array}\right]=\left[\begin{array}{ccc}
e^{\lambda_{1} t} & 0 & 0 \\
0 & e^{\lambda_{2} t} & 0 \\
l e^{\lambda_{1} t} m & e^{\lambda_{2} t} & e^{-\omega_{3} t}
\end{array}\right] .
$$

When $t=n T$, the linearization of (33) is

$$
\left[\begin{array}{l}
s\left(t^{+}\right) \\
r\left(t^{+}\right) \\
v\left(t^{+}\right)
\end{array}\right]=\left[\begin{array}{ccc}
1-\mu & 0 & 0 \\
0 & 1+p & 0 \\
0 & 0 & 1
\end{array}\right]\left[\begin{array}{l}
s(t) \\
r(t) \\
v(t)
\end{array}\right]
$$

The stability of the solution $\left(0,0, z^{*}\right)$ is determined by the eigenvalues of

$$
\Phi=\left[\begin{array}{ccc}
1-\mu & 0 & 0 \\
0 & 1+p & 0 \\
0 & 0 & 1
\end{array}\right] \phi(T)=\left[\begin{array}{ccc}
(1-\mu) e^{\lambda_{1} T} & 0 & 0 \\
0 & (1+p) e^{\lambda_{2} T} & 0 \\
l e^{\lambda_{1} t} m & e^{\lambda_{2} T} & e^{-\omega_{3} T}
\end{array}\right]
$$

We have

$$
\operatorname{det}(\Phi-\eta I)=\left|\begin{array}{ccc}
(1-\mu) e^{\lambda_{1} T}-\eta & 0 & 0 \\
0 & (1+p) e^{\lambda_{2} T}-\eta & 0 \\
l e^{\lambda_{1} t} & m e^{\lambda_{2} T} & e^{-\omega_{3} T}-\eta
\end{array}\right|=0
$$

where $\eta$ denotes the eigenvalues of $\Phi$, so that the three eigenvalues are

$$
\begin{aligned}
& \eta_{1}=(1-\mu) e^{\lambda_{1} T}, \\
& \eta_{2}=(1+p) e^{\lambda_{2} T},
\end{aligned}
$$

and

$$
\eta_{3}=e^{-\omega_{3} T}
$$

We have $\left|\eta_{1}\right|<1$ and $\left|\eta_{2}\right|<1$ since (40) holds, whereas $\left|\eta_{3}\right|<1$ since $\omega_{3}>0$. Hence, from the Floquet theory of impulsive differential equations, the solution $\left(0,0, z^{*}\right)$ is locally asymptotically stable.

We next investigate possible periodic solutions and their stability in the case that the nutrient is nonlimiting and there is enough supply of nutrient, so that its level remains at a constant level. 


\subsection{Nonlimiting nutrient}

To identify periodic solutions and their stability conditions, we will consider the case in which the nutrient is nonlimiting and its level has tended relatively quickly to a fixed equilibrium level $z=z_{0}$ at $E_{0}$, where it remains with

$$
\omega_{3} z^{*}-\frac{a_{3} x z_{0}}{K_{s}+z_{0}}-\frac{a_{4} y z_{0}}{K_{R}+z_{0}}-\omega_{3} z_{0}=0
$$

where $\frac{d z}{d t}=0$. For convenience, we let $x_{1}(t)=x(t), x_{2}(t)=y(t)$ and

$$
\begin{array}{ll}
\hat{a}_{1}=\frac{a_{1} z_{0}}{K_{S}+z_{0}}, & \hat{\psi}_{R}=\frac{\psi_{R} z_{0}}{K_{R}+z_{0}}, \\
\hat{\omega}_{1}=a_{2}+\omega_{1}, & \hat{\omega}_{2}=\omega_{1}-\hat{\psi}_{R} .
\end{array}
$$

With these substitutions, (32)-(33) are reduced to

$$
\begin{aligned}
& \left.\begin{array}{l}
\frac{d x_{1}}{d t}=\hat{a}_{1} x_{1}\left(\gamma-x_{1}\right)-\frac{\varepsilon_{r} x_{1}(t) x_{2}(t)}{k_{\gamma}+x_{1}(t)}-\hat{\omega}_{1} x_{1}, \\
\frac{d x_{2}}{d t}=\frac{\varepsilon_{r} e^{-\mu_{1} \tau} x_{1}(t-\tau) x_{2}(t-\tau)}{k_{\gamma}+x_{1}(t-\tau)}-\hat{\omega}_{2} x_{2}
\end{array}\right\}, \quad t \neq t_{n}=n T, n=1,2,3, \ldots, \\
& \left.\begin{array}{l}
x_{1}\left(t_{n}^{+}\right)=(1-r) x_{1}\left(t_{n}\right), \\
x_{2}\left(t_{n}^{+}\right)=(1+p) x_{2}\left(t_{n}\right)
\end{array}\right\}, \quad t_{n}=n T, n=1,2,3, \ldots
\end{aligned}
$$

We will first find a periodic solution to a reduced system of (43)-(44) under the assumption of nonlimiting nutrient and then use this solution to write a solution to the full system (43)-(44). We the will investigate the stability of these solutions. Accordingly, we first put $x_{1}(t) \equiv 0$ for all $t$ to obtain

$$
\begin{aligned}
& \frac{d x_{2}}{d t}=-\hat{\omega}_{2} x_{2}, \quad t \neq t_{n}, \\
& x_{2}\left(t_{n}^{+}\right)=(1+p) x_{2}\left(t_{n}\right), \quad t_{n}=n T, n=0,1,2, \ldots, \\
& x_{2}\left(0^{+}\right) \equiv x_{2_{0}} .
\end{aligned}
$$

Then we can easily see that a periodic solution of (45)-(46) is

$$
\tilde{x}_{2}(t)=(1+p)^{n} e^{-\hat{\omega}_{2}(t-n T)}, \quad n T<t<(n+1) T, n=0,1,2, \ldots,
$$

with $\tilde{x}_{2}\left(0^{+}\right)=1$, provided that

$$
(1+p) e^{-\hat{\omega}_{2} T}=1
$$

Thus a positive solution of (45)-(47) is

$$
\hat{x}_{2}(t)=\left(x_{2_{0}}-1\right) e^{-\hat{\omega}_{2} t}+\tilde{x}_{2}(t), \quad n T<t<(n+1) T, n=0,1,2, \ldots
$$

Therefore, if (48) holds, then system (43)-(44) has a periodic solution at the vanishing sensitive strain:

$$
\left(0, \tilde{x}_{2}(t)\right)=\left(0,(1+p)^{n} e^{-\hat{\omega}_{2}(t-n T)}\right), \quad(n-1) T<t<n T .
$$


Theorem 4 The solution $\left(0, \tilde{x}_{2}(t)\right)$ of (43)-(44) is locally asymptotically stable, provided that

$$
\begin{aligned}
& (1-r) \exp \int_{0}^{T}\left(a_{1} \gamma-\frac{\varepsilon_{r}}{k_{\gamma}} \tilde{x}_{2}(s)-\hat{\omega}_{1}\right) d s<1, \\
& (1+p) e^{-\hat{\omega}_{2} T}<1 .
\end{aligned}
$$

Proof Consider a small perturbation from the solution $\left(0, \tilde{x}_{2}(t)\right)$ :

$$
\begin{aligned}
& x_{1}(t)=u(t), \\
& x_{2}(t)=\tilde{x}_{2}(t)+v(t) .
\end{aligned}
$$

We will show that as time progresses, the perturbation from the solution $\left(0, \tilde{x}_{2}(t)\right)$ becomes smaller, and $(u(t), v(t)) \rightarrow(0,0)$, as long as the initial point for $(u(t), v(t))$ is close enough to $(0,0)$. The fundamental matrix of (43) satisfies

$$
\frac{d \Phi}{d t}=\left(\begin{array}{cc}
a_{1} \gamma-\frac{\varepsilon_{r}}{k_{\gamma}} \tilde{x}_{2}-\hat{\omega}_{1} & 0 \\
* & -\hat{\omega}_{2}
\end{array}\right) \Phi .
$$

Thus

$$
\Phi=\left(\begin{array}{cc}
\exp \int_{0}^{t}\left(a_{1} \gamma-\frac{\varepsilon_{r}}{k_{\gamma}} \tilde{x}_{2}(s)-\hat{\omega}_{1}\right) d s & 0 \\
* & \exp \left(-\int_{0}^{t} \hat{\omega}_{2} d s\right)
\end{array}\right),
$$

where it is not necessary to know the exact expression for $*$ since it does not come into later analysis.

Linearizing (44), we obtain

$$
\left(\begin{array}{l}
u\left(t^{+}\right) \\
v\left(t^{+}\right)
\end{array}\right)=\left(\begin{array}{cc}
1-r & 0 \\
0 & 1+p
\end{array}\right)\left(\begin{array}{l}
u(t) \\
v(t)
\end{array}\right)
$$

By the Floquet theory the stability of $\left(0, \tilde{x}_{2}(t)\right)$ depends on the eigenvalues of

$$
M_{0}=\left(\begin{array}{cc}
1-r & 0 \\
0 & 1+p
\end{array}\right) \Phi
$$

For asymptotic stability of $\left(0, \tilde{x}_{2}(t)\right)$, we need the multipliers to be smaller than 1 in absolute values, which is guaranteed by (49) and (50). Thus, by the Floquet theory of impulsive differential equations, $\left(0, \tilde{x}_{2}(t)\right)$ is locally asymptotically stable. This completes the proof.

We observe here that if $(50)$ holds, then $\left(0, \tilde{x}_{2}(t)\right)$ is not periodic since (48) is violated. Thus, if we assume (48), then one of the multipliers is equal to one, and we can only establish local stability, not asymptotical stability, of the periodic solution $\left(0, \tilde{x}_{2}(t)\right)$ in this case.

In Fig. 3, we present a numerical simulation of the model system (32)-(33) showing the jumps in the time series of bacterial populations every interval of $T=3$. Here the 
Figure 3 Time series solution under impulsive drug treatments and delay. Numerical solution of (32)-(33) where the sensitive and resistant strains undergo impulsive jumps every time drug treatment is applied. Here, $x(0)=0.3, y(0)=0.4, z(0)=0.5$, $a_{1}=2.407407, a_{2}=0.023077, a_{3}=0.00196$, $a_{4}=0.0007, \omega_{1}=0.7, \omega_{2}=0.7, \omega_{3}=0.7, K_{S}=0.7$, $K_{\gamma}=4.3, K_{R}=5.0, \varepsilon_{r}=0.9, \gamma=4.0, \mu=0.01, z^{*}=5.0$, $\tau=0.1$ and $\psi_{R}=0.5, r=0.1, p=1.0, T=3$

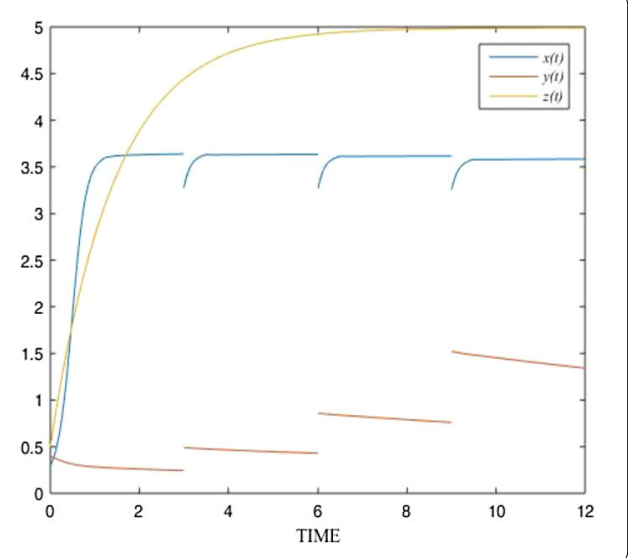

Figure 4 Controlled resistance under impulses and delay. Time series solution of (32)-(33) where the resistant strain remains under a controllable level. $x(0)=0.3, y(0)=0.4, z(0)=0.5, a_{1}=2.407407$, $a_{2}=0.023077, a_{3}=0.00196, a_{4}=0.0007, \omega_{1}=0.7$, $\omega_{2}=0.7, \omega_{3}=0.7, \varepsilon_{r}=0.9 K_{S}=0.7, K_{\gamma}=4.3, K_{R}=5.0$, $\gamma=4.0, \mu=0.01, z^{*}=5.0, \tau=0.1$ and $\psi_{R}=0.5$, $r=0.1, p=0.1, T=3$

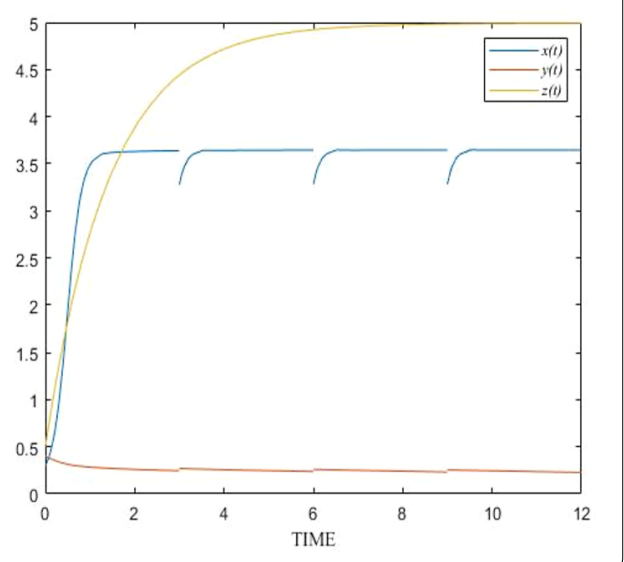

multiplication factor $p$ is high, in which case the resistant strain is seen here to increase unboundedly as time passes. In Fig. 4 , on the other hand, $p$ is small relative to $T$, so that (38) is satisfied, and the resistant strain in kept to a low level $z_{0}$. Beyond the point where $z$ has reached $z_{0}$, the system can be modeled by (43)-(44).

\section{Discussion and conclusion}

We have analyzed a model of microbial resistance to drugs to investigate how the dynamic behavior of the system may be affected by a delay in the process of plasmid transfer. Specifically, the existence of sustained oscillations in solutions of the model has been of interest, since such oscillatory behavior has been commonly observed in clinical data. We have found that under suitable conditions, a Hopf bifurcation can occur leading to limit cycles emanating from the endemic equilibrium solution, ensuring that the infection is endemic but fluctuates periodically.

Since resistance is expected if prolonged drug use is the practice, we investigate whether this can be managed in a more efficient manner if deeper understanding is gained. The base model has been extended to incorporate impulsive drug prescription, which, apart from killing a fraction of the sensitive bacterial strain, also allows the resistant strain to benefit from the abundance of nutrients to multiply in periodic bursts at the same time. This model system (32)-(33) is shown to possess an equilibrium solution, where the bacterial strains are washed out, which is locally asymptotically stable, provided that inequal- 
ities (34) and (37) are satisfied. Suppose we define the basic reproductive number $R_{0}$ as

$$
R_{0}=\max \left\{\frac{\psi_{R} z^{*}}{\omega_{2}\left(K_{R}+z^{*}\right)}, \frac{\left(a_{2}+\omega_{1}\right)\left(K_{S}+z^{*}\right)}{a_{1} \gamma z^{*}}\right\} .
$$

Then, if the density of each of the bacterial strains does not start out from too high a value, then bacterial infection will be eradicated, provided that

$$
R_{0}<1
$$

and

$$
T_{1}>T>T_{2}
$$

with $T_{1}$ and $T_{2}$ given in (34) and (35). That is, the local stability of the washout equilibrium can be ensured under suitable conditions.

Condition (51) is satisfied if the resistant bacterial strain removal rate $\omega_{2}$ is sufficiently high, or the half saturation constant $K_{R}$ is large enough, or $\psi_{R}$ and killing rate of the sensitive strain $a_{1}$ are relatively high compared to $\omega_{1}, a_{2}$, or $K_{S}$.

Condition (52) can be accomplished if the interval between drug intakes is in the proper range. If the fraction $p$ at which the resistant bacterial strain can multiply itself taking the advantage of deaths in the sensitive strain due to drug intakes is too high, then we see that this condition (52) will not be achievable as $T_{2}$ could be bigger than $T_{1}$ so that we would not be able to find $T$ between $T_{1}$ and $T_{2}$ that satisfies (52), in which case the bacterial strains may diverge away from the washout equilibrium state.

Now, $p$ reflects how efficiently the resistant bacteria can multiply in bursts, each time drug treatment is applied (because sensitive bacteria are killed off making the resources more abundant for the resistant strain). So, if $p$ is relatively high, then $T$ must not be too small, because the bursts in resistant bacterial growth will be too frequent, resulting in the environment being overgrown with resistant strain. Namely, if we keep $T$ long enough so that $T>T_{2}$ (preventing resistant strain from bursts of growth that are too frequent) but short enough so that $T<T_{1}$ (killing off the sensitive bacteria frequently enough), then the bacterial populations can be eradicated, provided that their levels are not too high to begin with.

From our discussion we can see that mathematical models construction and analyses can provide insights into the outcomes of interventions under a set of underlying structural and procedural assumptions. Modeling different mechanisms involving within-host competition permits the considerations of different intervention protocols and targets. The presence of strain coexistence, types of infection, resource limitation and availability, strain conversion, and delays affects the choice of model structure. With increasing concern about the spread of antibiotic resistance, the use of mathematical models should be made to yield valuable insights and better understanding of the mechanisms behind this spread. 
Competing interests

The authors declare that they have no competing interests.

\section{Authors' contributions}

The first author contributed to the bifurcation analysis and model simulations, the second and third authors contributed to the impulsive model analysis and manuscript production. All authors read and approved the final manuscript.

\section{Publisher's Note}

Springer Nature remains neutral with regard to jurisdictional claims in published maps and institutional affiliations.

Received: 29 January 2019 Accepted: 24 June 2019 Published online: 05 July 2019

\section{References}

1. Barai, L., Saha, M.R., Rahman, T., Khandaker, T., Dutta, S., Hasan, R., Haq, J.A.: Antibiotic resistance: situation analysis in a tertiary care hospital of Bangladesh. Banglad. J. Microbiol. 34, 15-19 (2017)

2. World Health Organisation (WHO): Antimicrobial resistance: global report on surveillance (2014) http://apps.who.int/iris/bitstream/10665/112642/1/9789241564748_eng.pdf

3. Zellweger, R.M., Carrique-Mas, J., Limmathurotsakul, D., Day, N.P.J., Thwaites, G.E., Baker, S.: A current perspective on antimicrobial resistance in Southeast Asia. J. Antimicrob. Chemother. 72, 2963-2972 (2017)

4. Spicknall, I.H., Foxman, B., Marrs, C.F., Eisenberg, J.N.S.: A modeling framework for the evolution and spread of antibiotic resistance: literature review and model categorization. Am. J. Epidemiol. 178(4), 508-520 (2013). https://doi.org/10.1093/aje/kwt017

5. Puttasontiphot, T., Lenbury, Y., Rattanakul, C., Rattanamongkonkul, S., Hotchkiss, J.R., Crooke, P.S.: Dynamic processes permitting stable coexistence of antimicrobial resistant and non-resistant organisms in a gastrointestinal tract model Science Asia 33, 197-206 (2007)

6. Chayapham, P., Lenbury, Y.: Stability and persistence of delayed resistant and sensitive bacterial strains interaction under impulsive drug treatment. Int. J. Math. Comput. Simul. 7, 475-484 (2013)

7. Andrup, L., Smidt, L., Andersen, K., Boe, L.: Kinetics of conjugative transfer: a study of the plasmid pXO16 from Bacillus thuringiensis subsp. Plasmid 40, 30-43 (1998)

8. Dostal, L., Shao, S., Schildbach, J.F.: Tracking F plasmid Tral relaxase processing reactions provides insight into F plasmid transfer. Nucleic Acids Res. 39, 2658-2670 (2011)

9. Sirinukunwattana, K., Lenbury, Y., Tumrasvin, N.: Drug resistant and wild-type strains interaction: investigating effects of conversion delays for possible control strategies. Int. J. Math. Models Methods Appl. Sci. 4, 830-838 (2011)

10. Rodríguez, G.: Lecture Notes on Generalized Linear Models (2007). http://data.princeton.edu/wws509/notes/

11. Culshaw, R.V., Ruan, S.: A delay-differential equation model of HIV infection of CD4+ T-cells. Math. Biosci. 165, 27-39 (2000)

12. Wang, W., Chen, L.: Stability and Hopf bifurcation analysis of an epidemic model by using the method of multiple scales. Math. Probl. Eng. 2016, Article ID 2034136 (2016). https://doi.org/10.1155/2016/2034136

13. Lakshmikantham, V., Bainov, D.D., Simeonov, P.S.: Theory of Impulsive Differential Equations. World Scientific, Singapore (1989)

\section{Submit your manuscript to a SpringerOpen ${ }^{\odot}$ journal and benefit from:}

- Convenient online submission

- Rigorous peer review

- Open access: articles freely available online

- High visibility within the field

- Retaining the copyright to your article 